\title{
Preliminary results of rocket attitude and auroral green line emission rate in the DELTA campaign
}

\author{
Naomoto Iwagami ${ }^{1}$, Sayaka Komada ${ }^{1}$, and Takao Takahashi ${ }^{2}$ \\ ${ }^{1}$ Department of Earth and Planetary Science, University of Tokyo, Bunkyo-ku, Tokyo 113-0033, Japan \\ ${ }^{2}$ Information Science Laboratory, Tokai University, Kitakaname, Hiratsuka 259-1292, Japan
}

(Received September 26, 2005; Revised June 23, 2006; Accepted July 12, 2006; Online published September 29, 2006)

\begin{abstract}
The attitude of a sounding rocket launched in the DELTA (Dynamics and Energetics of the Lower Thermosphere in Aurora) campaign was determined with IR horizon sensors and geomagnetic sensors. Since the payload was separated into two portions, two sets of attitude sensors were needed. A new IR sensor was developed for the present experiment, and found the zenith-angle of the spin-axis of the rocket with an accuracy of $2^{\circ}$. By combining information obtained by both type of sensors, the absolute attitudes were determined. The auroral green line emission rate was measured by a photometer on board the same rocket launched under active auroral conditions, and the energy flux of the auroral particle precipitation was estimated.
\end{abstract}

Key words: Rocket attitude, horizon sensor, geomagnetic sensor, auroral green line.

\section{Introduction}

In the data processing for a rocket experiment, it is usually needed to know the attitude of the rocket. For example, the main instrument of the present campaign, NTV (nitrogen temperature of vibration; Kurihara et al., 2003), needs such information because it is an in-situ measurement, which may be affected by the shock-wave structure produced around the rocket body. The density and temperature in the shock-wave region are disturbed, and need correction to know their natural values. Since the shape and the character of the shock-wave region depend on the rocket speed, atmospheric parameters such as density and temperature and the attitude of the rocket, information about the rocket attitude is important for a meaningful interpretation. Also for the optical remote sensing such as the airglow or aurora measurements, the attitude of the rocket is needed to correct geometrical effects; a slant ray path makes the apparent emission rate larger.

Although a sort of star-sensor is usually used for a nighttime rocket experiment, IR horizon sensors were chosen for the present experiment because of the auroral contaminations. Although stars of the 3rd magnitude are needed for the analysis of a star-sensor, they may not be seen in presence of the aurora. The rocket was separated into mother and daughter payloads at $100 \mathrm{sec}$ after the launch at 107 $\mathrm{km}$ in ascent. This is to keep the plasma and optical measurements on the mother payload away from the electrical and optical disturbances due to NTV on the daughter payload. NTV emits a beam of $100 \mathrm{eV}$ electrons, and could be a serious source of electrical and optical contaminations.

The lines of sight of the IR sensors were set a little downward so that they intersect the horizon when the rocket is

Copyright (c) The Society of Geomagnetism and Earth, Planetary and Space Sciences (SGEPSS); The Seismological Society of Japan; The Volcanological Society of Japan; The Geodetic Society of Japan; The Japanese Society for Planetary Sciences; TERRAPUB spinning. It is possible to determine the zenith angle of the rocket spin-axis from the duration to see the Earth within one spin cycle. On the other hand, geomagnetic sensors on board the mother and daughter payloads determined the angle between the rocket spin-axis and the geomagnetic line of force (hereafter called mag-angle). However, both sensors can only determine angles from certain references, and cannot find the absolute attitude. It may be obtained by combining information from the both attitude sensors.

The auroral green line $(557.7 \mathrm{~nm})$ is one of the most prominent features in the auroral visible spectrum. It has long been used to estimate various auroral parameters. For example, the energy flux of the precipitating particles may be estimated from the apparent emission rate, and the characteristic energy may be from the height distribution of the volume emission rate. The latter may be measured by a photometer on board a rocket penetrating the emitting region. However, this procedure needs an assumption of stable aurora.

In the present paper, the design, performance and accuracy of the new IR attitude sensor, the preliminary results of the attitudes of both portions of the payload, measurement of the auroral green line volume emission rate distribution, and estimation of the energy flux of the precipitating particles are presented.

\section{Instrumentation}

The IR radiometer on board the daughter payload, HOS (horizon sensor; Nakamura et al., 1989), measured the thermal emission at the $\mathrm{CO}_{2} 15 \mu \mathrm{m}$ band. Another one on board the mother payload, SFF (surface finder), measured the thermal emission in the $8-12 \mu \mathrm{m}$ atmospheric window region. HOS was developed by a company, and has been used for many satellite programs. However, we developed SFF because a stable supply of HOS was not expected, and a simplified (cheaper) version of HOS was needed for the 


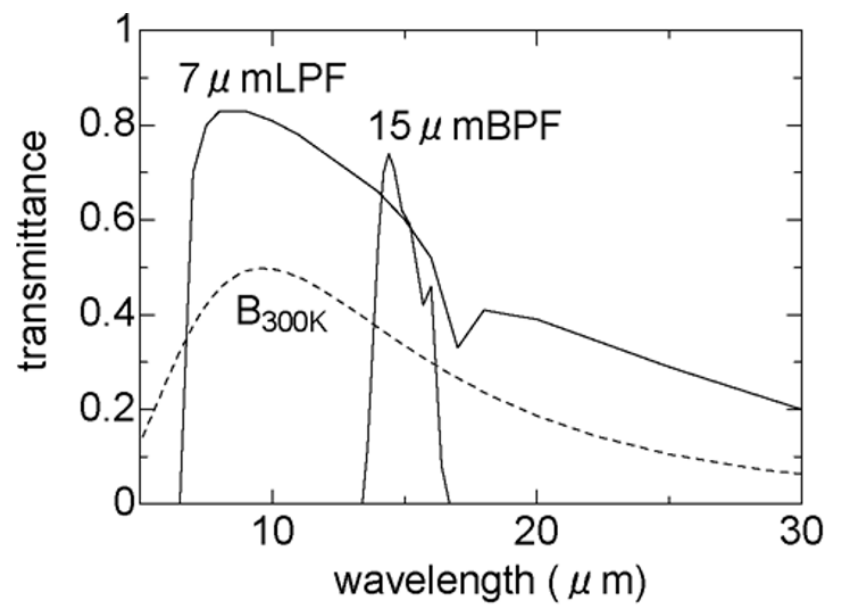

Fig. 1. Filter transmissions: $15 \mu \mathrm{m} \mathrm{BPF} \mathrm{(band-pass} \mathrm{filter)} \mathrm{for} \mathrm{HOS} \mathrm{and} 7$ $\mu \mathrm{m} \mathrm{LPF}$ (long-pass filter) for SFF. Relative shape of blackbody function of $300 \mathrm{~K}$ is also shown.

rocket experiment. Each radiometer consists of a Ge lens, a filter and a pyroelectric IR detector. This sort of detector is suitable for the rocket experiment because it works under the room temperature without any cooling system. HOS is designed to find out the $\mathrm{CO}_{2}$ horizon with a band-pass filter centered at $15 \mu \mathrm{m}$ with a FWHM of $2 \mu \mathrm{m}$. Since the optical thickness of the atmosphere in this wavelength region is so large due to $\mathrm{CO}_{2}$ absorption that the Earth's surface cannot be seen from the rocket, for example, at $100 \mathrm{~km}$. According to a radiative transfer calculation, the $\mathrm{CO}_{2}$ horizon seems to be located at around $37.0 \mathrm{~km}$ in the present experiment (see Appendix). On the other hand, SFF was designed to detect the solid Earth horizon with a $7 \mu \mathrm{m}$ long-pass filter. This wavelength selection comes from the fact that a one order of magnitude larger input signal than that for HOS was expected; that is, 10 times larger $\mathrm{S} / \mathrm{N}$ ratio may be obtained as far as the optical and the electrical systems are similar. According to a radiative transfer calculation shown in Appendix, the apparent horizon height is found to be $5.7 \mathrm{~km}$ for SFF. In Fig. 1 the filter transmissions are shown with relative shape of the $300 \mathrm{~K}$ blackbody function. The field of view of HOS and SFF are $1.3^{\circ}$ and $1.0^{\circ}$ full angle, respectively, and their lines of sight were set $103.8^{\circ}$ and $105.0^{\circ}$, respectively, away from the spin-axis. These offset angles are important, and are results of optimization. A too large angle such as $120^{\circ}$ may cause no horizon crossing, and a too small angle such as $100^{\circ}$ may result little sensitivity in the solution. Since the rocket is usually spinning with a period of about $1 \mathrm{sec}$ during the observation, the lines of sight of HOS and SFF usually cross the horizon twice a second. Since the portion to see the Earth varies with the zenith angle of the spin-axis, it can be used to estimate the spin-axis zenith angle.

Figure 2 shows examples of the relation between the spin-axis zenith angle and the portion of the Earth seen in one spin period. Note that the relation also varies with rocket height. As seen in the figure, almost no crossing occurs if the spin-axis zenith angle is less than $5^{\circ}$. On the other hand, the output becomes insensitive to the change in zenith angles if it is larger than $25^{\circ}$. It appears to be

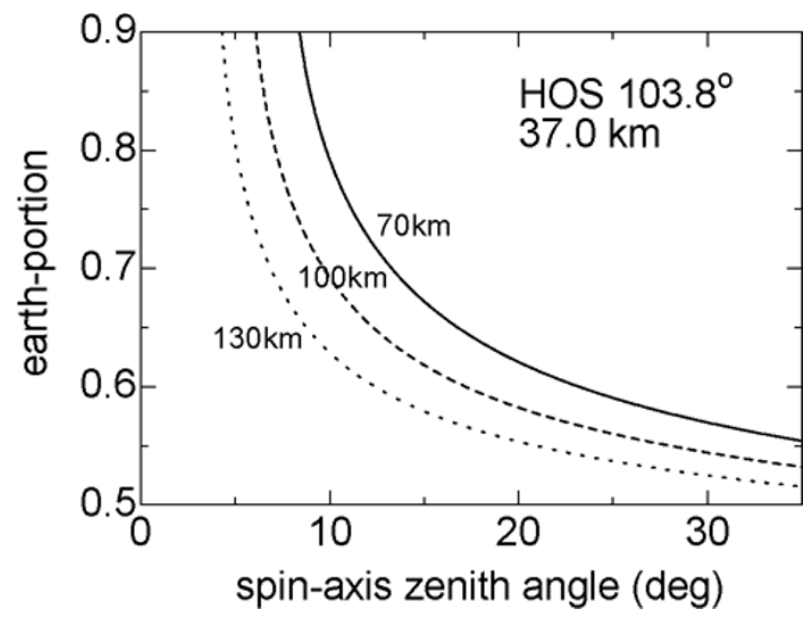

Fig. 2. Examples of the relation between the spin-axis zenith angle and the portion of the Earth seen in one spin period for 3 rocket heights of 70,100 and $130 \mathrm{~km}$.

important to adjust the sensitive angle region to that to be measured. The output of SFF is expected to be larger than that of HOS by about 10 times (see Appendix) because it measures Earth's radiance in the atmospheric window region. This is an advantage of SFF over HOS. On the other hand, HOS has an advantage that it can be used without suffering disturbances from the cloud, which may not be distributed uniformly. The accuracy in the spin-axis zenith angles by HOS and SFF for the present rocket experiment is estimated to be $2^{\circ}$. Since the error in determining the Earthportion is $1 \%$ (due to $0.01 \mathrm{sec}$ uncertainty in the crossing time), which corresponds to about $2^{\circ}$ in the spin-axis zenith angle according to the relation seen in Fig. 2.

The GA (geomagnetic aspectmeter; Takahashi and Tohyama, 1998) on board the daughter payload is a double axial fluxgate magnetometer with ring core sensors. It has a dynamic range of $\pm 50,000 \mathrm{nT}$ and a sensitivity of 380 $\mathrm{nT} / \mathrm{LSB}$ for each axial component. The $\mathrm{Z}$ axis of the sensor was aligned to the rocket spin axis and the $H$ axis was located in a plane perpendicular to the spin axis. The $\mathrm{Z}$ and $\mathrm{H}$ components of the geomagnetic field in the rocket coordinate system were measured every $2.5 \mathrm{msec}$ with a noise level less than $380 \mathrm{nT}$. The GA can determine the mag-angle with an accuracy of $1^{\circ}$. It can also measure the spin frequency of the rocket. The GA on board the mother payload has a dynamic range of $\pm 100,000 \mathrm{nT}$ and a sensitivity of $49 \mathrm{nT} / \mathrm{LSB}$ for each axial component. The $\mathrm{Z}$ axis of the sensor was aligned to the rocket spin axis, and the $\mathrm{X}$ and $\mathrm{Y}$ axes were located in a plane perpendicular to the spin axis. Three components of the geomagnetic field in the rocket coordinate system were measured every 1.25 msec with a noise level less than $200 \mathrm{nT}$.

AGL (auroral green line photometer; Iwagami et al., 2003) on board the mother payload consists of a bandpass filter, a lens and a PMT (photomultiplier tube). The band-pass filter has a central wavelength of $557.7 \mathrm{~nm}$ and a FWHM of $6.2 \mathrm{~nm}$. The field of view is a circle with a diameter of $6^{\circ}$. The PMT sensitive in the $160-650 \mathrm{~nm}$ region is set in a small air-tight room to prevent it from discharging. The line of sight was set in the direction $60^{\circ}$ away from 


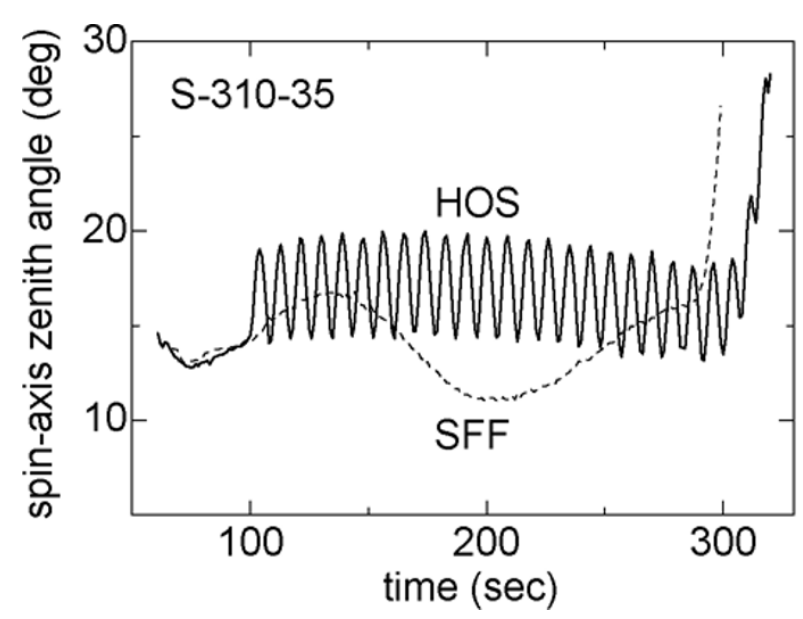

Fig. 3. Spin-axis zenith angles of the daughter and mother payloads determined by HOS (real curve) and SFF (dashed curve), respectively. SFF data coincide within $1^{\circ}$ to HOS data before separation at $100 \mathrm{sec}$.

the spin-axis. Usually, a smaller offset angle such as $30^{\circ}$ is preferred; however, the configuration of the mother payload did not allow it.

\section{Rocket Experiment}

The sounding rocket S-310-35 was launched at 00:33 UT on 13 December 2004 from Andoya Rocket Range $\left(69^{\circ} \mathrm{N}\right.$, $16^{\circ} \mathrm{E}$ ) in the direction of $81^{\circ}$ in elevation and $355^{\circ}\left(5^{\circ}\right.$ west of North) in azimuth. The optical measurements started after opening a nose faring of the daughter payload at 60 $\mathrm{sec}$ at $67 \mathrm{~km}$ (opening of a door of the mother payload was at $62 \mathrm{sec}$ at $70 \mathrm{~km}$ ), and the separation was taken at 100 sec after the launch at $107 \mathrm{~km}$. Both payloads reached the maximum height of $140 \mathrm{~km}$ at $184 \mathrm{sec}$, came down to 100 $\mathrm{km}$ at $276 \mathrm{sec}$, and fell on the sea. Although the initial plan was to launch the rocket into a stable diffuse aurora, the actual launch was carried out under the brake-up-like active auroral conditions. The direction of the magnetic line of force is $77.8^{\circ}$ in elevation and $183.8^{\circ}$ in azimuth at the rocket range.

\section{Results}

Figure 3 shows results of HOS and SFF measurements. It is seen in the figure that the daughter payload started a fast precession with a period of $8.7 \mathrm{sec}$ just after the separation at $100 \mathrm{sec}$. On the other hand, the mother payload kept a usual precession with a period of $150 \mathrm{sec}$. At the end of the flight, the attitude of the mother payload began to change due to air drug at $290 \mathrm{sec}$ around $87 \mathrm{~km}$ in descent as a usual S-310 flight. Whereas, that of the daughter payload started to change later at $300 \mathrm{sec}$ around $77 \mathrm{~km}$. Probably, this difference is due to shorter and more symmetrical shape of the daughter payload than the mother payload. Note that the results of both HOS and SFF coincide within $1^{\circ}$ before the separation. This suggests that both sensors have accuracy better than $2^{\circ}$ as claimed before.

Figure 4 shows the mag-angles obtained by GAH on board the daughter payload and by GAX on board the mother payload. In the present work, the mag-angles were calculated under the following assumptions: (1) the attitude

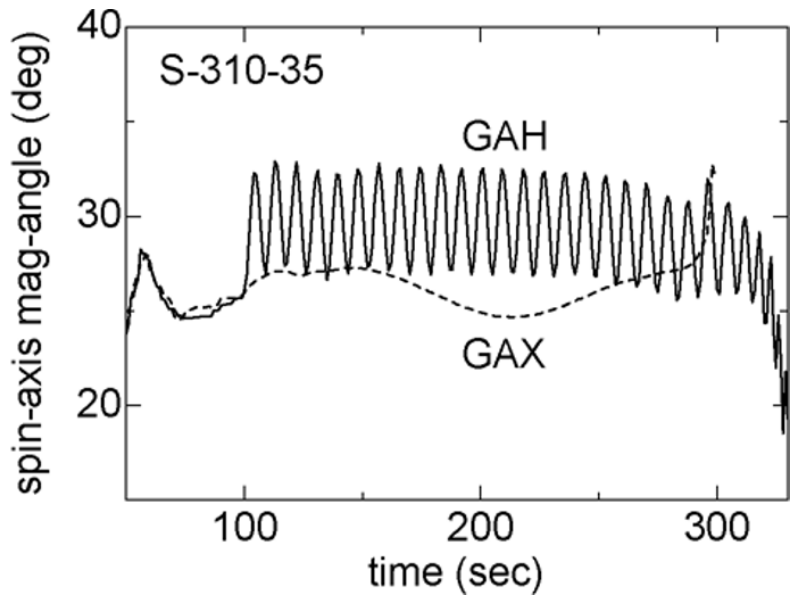

Fig. 4. Angles between the spin-axis and the geomagnetic line of force (mag-angles) measured by GAH and GAX on board the daughter and mother payloads, respectively.

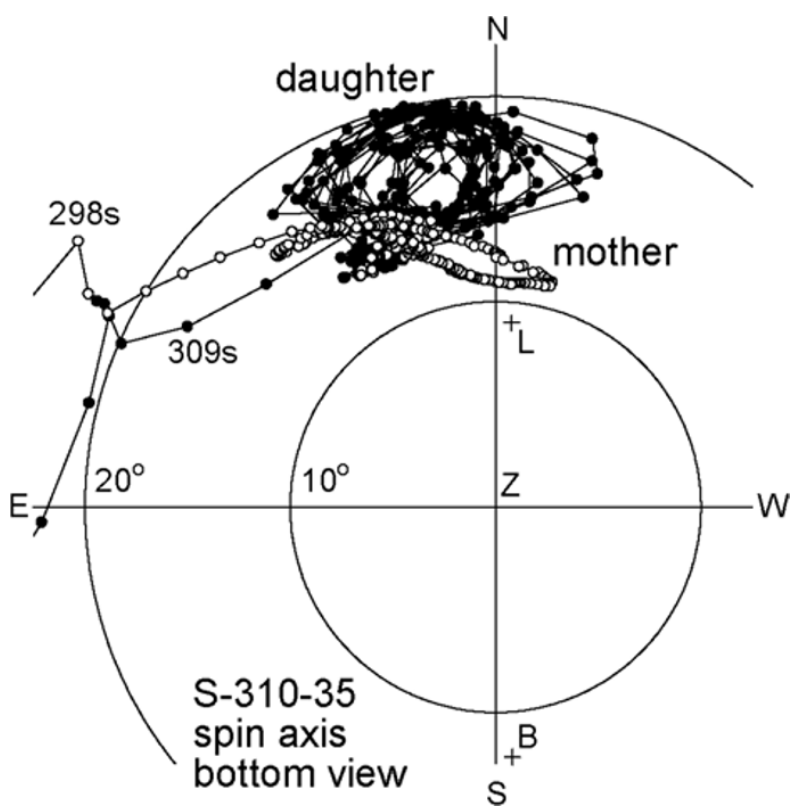

Fig. 5. Spin-axis directions of the daughter (line with dot) and mother (line with open circle) payloads plotted every second in the sky around the zenith. For the daughter and mother payloads, data points of 61-315 $\mathrm{sec}$ and 67-298 sec, respectively, are plotted. The launching direction (L) and the direction of the magnetic line of force (B) are indicated by pluses $(+)$.

just after the launch was the same as on the launcher, (2) the attitude just after the separation was the same as just before the separation, it means that the changes of data just after the separation were caused by only magnetic bias from rocket body, and (3) the total geomagnetic line of forces measured using with GAX and GAH were the same as the reference magnetic filed along the rocket trajectory, which was computed using the IGRF model (IAGA Division V Working Group VMOD., 2005). Unfortunately, the GAZ of the mother payload was saturated by the magnetic bias from the rocket body after the separation. Therefore, the mag-angles were calculated by the data of GAH and the total geomagnetic line of forces computed using the IGRF model after the separation. As a natural consequence, the 


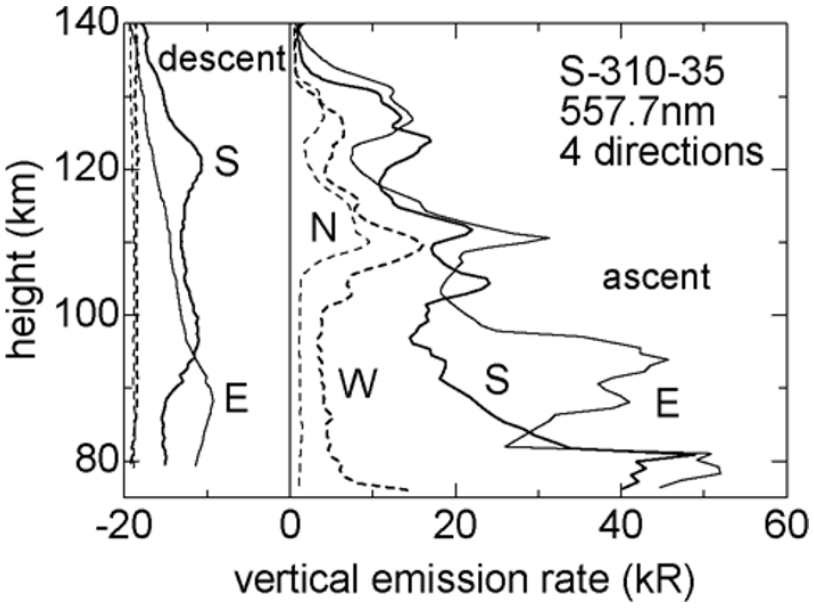

Fig. 6. Auroral green line $(557.7 \mathrm{~nm})$ vertical emission rates sorted into 4 directions: north (thin dashed curve), east (thin real curve), south (thick real curve) and west (thick dashed curve). The descent data are shifted toward the left by $20 \mathrm{kR}$.

mag-angles by GAH and GAX show similar behavior as the spin-axis zenith angles by HOS and SFF, respectively.

By combining information obtained by the horizon and magnetic sensors, it is possible to solve the absolute direction of the spin-axis (Tohyama et al., 1988). This is a procedure to find out 2 intersecting points of 2 small circles in the sky. There appear two sets of solutions, and the one showing a clockwise precession is the true solution because the spinning motion is clockwise. Figure 5 shows the directions of spin-axis of the mother and daughter payloads plotted every second in the sky around the zenith. It is found in the figures that both spin-axis directions drew precession circles in the northern part of the sky. However, their shapes (especially that of the mother payload) appear to be unnatural being far different from a simple small circle. This probably means that considerable error is still included in the results of HOS, SFF, GAH or GAX. By comparing Figs. 3 and 4 , it is found that the amplitudes of the precessing motion of the mother payload after the separation differs each other considerably (about 5 degree by SFF and 2 degree by GAX).

In Fig. 6 the vertical emission rates of the auroral green line sorted into four directions are plotted versus the rocket height. Since the apparent emission rate is spin-modulated considerably, only spin-averaged values are shown. Due to serious spatial inhomogeneity especially during the ascent, the emission rates in the 4 directions show complicated behaviors. Among them, those in the east and south directions appear to be brighter than the others at heights below $110 \mathrm{~km}$ in ascent. This is consistent to the map projections of aurora arcs over Andoya imaged at Kilpisjarvi, Finland (Kauristie and Ogawa, private communication; also see Kurihara et al., this issue). They show a bright arc located east and south of Andoya during ascent. During descent the auroral structure became diffuser than in ascent, and both time and spatial variation became calm.

From these volume emission rate profiles in the ascent and descent, it may possible to estimate the precipitating energy flux by referring a simulation of Banks et al. (1974) and the emission height information from the ground-based measurement. According to the latter, the height of the emitting layer was estimated to be $120 \mathrm{~km}$ (see Kurihara et al., this issue). With this height and the calculated green line volume emission rate for unit incident flux shown in figure 12 of Banks et al. (1974), the characteristic energy of the precipitating auroral electrons is estimated to be 4 $\mathrm{keV}$. The emission efficiency was obtained by integrating the volume emission rate profile to be $150 \mathrm{R} /\left(\mathrm{erg} \mathrm{cm}^{-2} \mathrm{~s}^{-1}\right)$. With this emission efficiency, it is possible to estimate the energy flux from the measured apparent emission rates. As seen in Fig. 6 in ascent below $100 \mathrm{~km}$, the mean vertical emission rates in the south and east directions are about $30 \mathrm{kR}$, and those in the north and west directions were 3 $\mathrm{kR}$; the precipitating energy fluxes are estimated to be 200 and $20 \mathrm{erg} \mathrm{cm}^{-2} \mathrm{~s}^{-1}$, respectively. And in descent below $100 \mathrm{~km}$, the former was about $8 \mathrm{kR}$ and the latter was 1.5 $\mathrm{kR}$; the fluxes are estimated to be 50 and $10 \mathrm{erg} \mathrm{cm}^{-2} \mathrm{~s}^{-1}$, respectively.

\section{Summary}

The attitudes of the both payloads of the rocket in the DELTA campaign were determined with IR horizon sensors and geomagnetic sensors. After the separation at $100 \mathrm{sec}$, the daughter payload started an unusually fast precession with a period of $8.7 \mathrm{sec}$, whereas the mother payload kept a typical precessing motion with a period of $150 \mathrm{sec}$. The newly developed IR attitude sensor SFF could determine the spin-axis zenith angle with an accuracy of 2 degree. The precipitating energy fluxes were estimated to be 200 and 50 erg $\mathrm{cm}^{-2} \mathrm{~s}^{-1}$ for the east and south directions during ascent and descent, respectively.

Acknowledgments. The authors thank to the rocket launching clue of the Institute of Space and Astronautical Science and the people at Andoya Rocket Range for their support to complete a successful experiment.

\section{Appendix.}

It is needed to know the apparent height of the horizon for

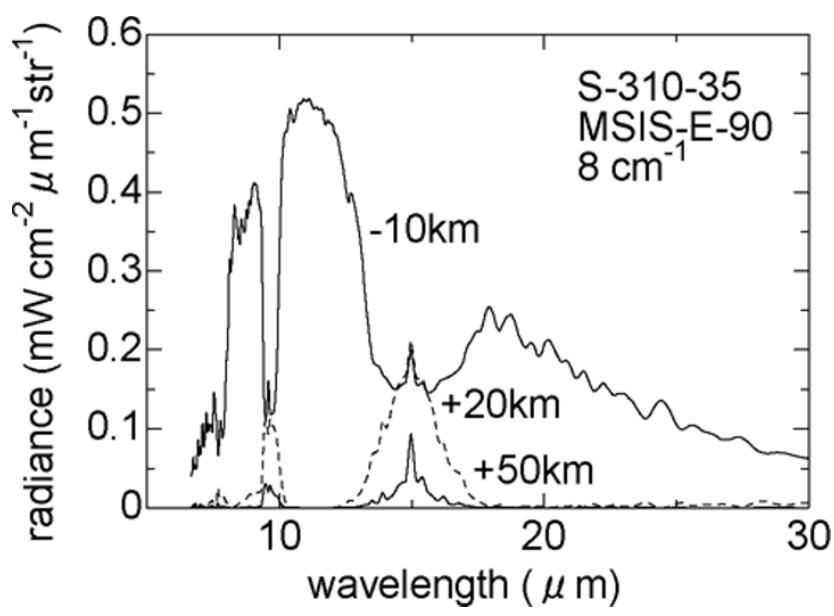

Fig. A.1. Examples of thermal radiation spectrum for tangent heights of $-10 \mathrm{~km}$ (thick real curve), $+20 \mathrm{~km}$ (dashed curve) and $+50 \mathrm{~km}$ (thin real curve) calculated using HITRAN2000 molecular database and the MSIS-E-90 model after a smoothing with a FWHM of $8 \mathrm{~cm}^{-1}$. 


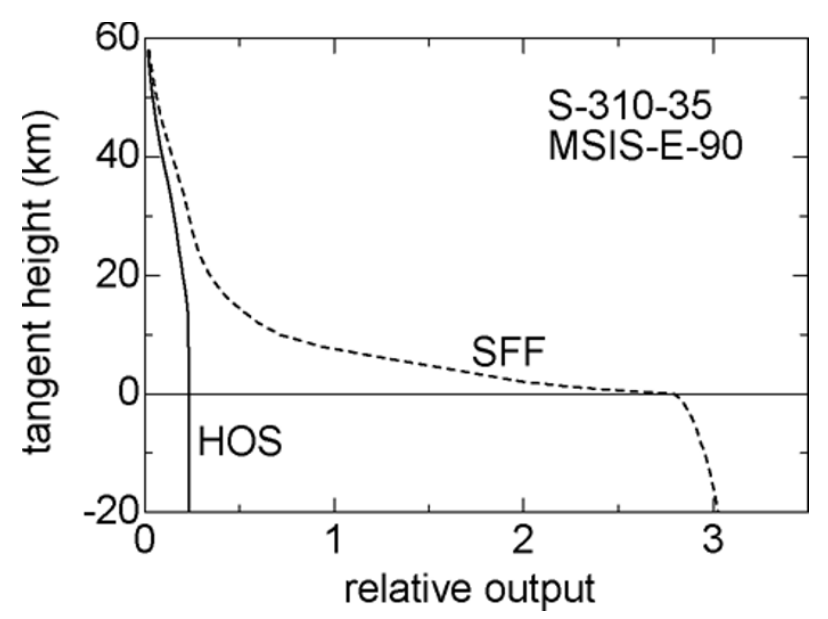

Fig. A.2. Expected outputs of HOS and SFF as functions of tangent height of the line of sight. The half-maxima occur at $37.0 \mathrm{~km}$ and $5.7 \mathrm{~km}$ for HOS and SFF, respectively.

the particular wavelength region by taking the actual spectrum of the Earth's thermal radiation into account. For example, the wavelength region used for HOS is located in a strong $\mathrm{CO}_{2}$ band, and the solid surface cannot be seen from the rocket. The apparent $\mathrm{CO}_{2}$ horizon height must be estimated by considering the actual atmospheric temperature structure and distributions of minor but IR active constituents such as $\mathrm{O}_{3}$. Since SFF uses the atmospheric window region, the solid surface may be seen. However, the actual height of the horizon may differ from $0 \mathrm{~km}$ if actual atmospheric conditions are considered. In this Appendix, calculations of those apparent horizon heights are described.

The thermal emission of the Earth and atmosphere may be calculated as (e.g. Schandra, 1986)

$$
I=B_{0} T_{0}+\int_{\text {LOS }} B(s) \frac{d T(s)}{d s} d s
$$

with

$$
T(s)=\exp \left[-\sum_{i} \sigma_{i}(s) N_{i}(s)\right],
$$

where $B_{0}$ is black-body function of the surface temperature; $T_{0}$ is transmission from the surface to the observer; $B$ is black-body function; $T(s)$ is transmission from $s$ to the observer; $s$ is path length; $\sigma_{i}$ is absorption cross section of gas $i, N_{i}(s)$ is the column density of gas $i$ between $s$ and the observer; and the integration in Eq. (A.1) should be done along the line of sight (LOS). If it does not hit the surface, the first term $B_{0} T_{0}$ must not be taken into account. The second term represents the contribution from the atmosphere. To calculate the transmission $T(s)$, a molecular spectroscopic database HITRAN2000 and a model atmosphere MSIS-E-90 were used. The gases taken into account are $\mathrm{CO}_{2}, \mathrm{H}_{2} \mathrm{O}, \mathrm{O}_{3}, \mathrm{CH}_{4}$ and $\mathrm{N}_{2} \mathrm{O}$. In Fig. A.1 the spectral radiance seen from the rocket for the tangent heights of $-10 \mathrm{~km},+20 \mathrm{~km}$ and $+50 \mathrm{~km}$ are shown as examples. A positive tangent height means that the line of sight does not hit the surface, and only the atmosphere contributes to the radiance. In case of negative tangent height of $-10 \mathrm{~km}$, both the surface and the atmosphere contribute. The upper envelope of the top spectrum is close to a blackbody of the surface temperature.

The expected output for HOS and SFF may be calculated from such spectra and the filter transmissions shown in Fig. 1. In Fig. A.2 the expected output of HOS and SFF are plotted versus tangent height. It is seen in the figure, the half-maximum values appear at $37.0 \mathrm{~km}$ and $5.7 \mathrm{~km}$ for HOS and SFF, respectively. The output for SFF is about 10 times larger than that of HOS as expected.

\section{References}

Banks, P. M, C. R. Chappell, and A. F. Nagy, A new model for the interaction of auroral electrons with the atmosphere: Spectral degradation, backscatter, optical emission, and ionization, J. Geophys. Res., 79, 1459-1470, 1974.

HITRAN2000, http://cfa-www.harvard.edu/hitran//

IAGA Division V, Working Group VMOD., The 10th Generation International Geomagnetic Reference Field, Geophys. J. Int., 161, 561-565, 2005.

IGRF, http://swdcdb.kugi.kyoto-u.ac.jp/igrf/point/index-j.html.

Iwagami, N., T. Shibaki, T. Suzuki, H. Sekiguchi, and N. Takegawa, Rocket observation of atomic oxygen density and airglow emission rate in the WAVE2000 campaign, J. Atm. Solar-Terr. Phys., 65, 1349-1360, 2003.

Kurihara, J., K-I. Oyama, K. Suzuki, and N. Iwagami, Vibrationalrotational temperature measurement of $\mathrm{N}_{2}$ in the lower thermosphere by the rocket experiment, Adv. Space Res., 32(5), 725-729, 2003.

Kurihara, J., T. Abe, K. Oyama, E. Griffin, M. Kosch, A. Aruliah, K. Kauristie, Y. Ogawa, S. Komada, and N. Iwagami, Observations of the lower thermospheric neutral temperature and density in the DELTA campaign, Earth Planets Space, 58, this issue, 1123-1130, 2006.

MSIS-E-90 Atmosphere Model, http://modelweb.gsfc.nasa.gov/models/ msis.html.

Nakamura, K., T. Ishigaki, A. Kaneko, S. Takahashi, J. Nishida, Y. Wakabayashi, and H. Nakamura, Pyroelectric infrared detector for precision Earth sensor, International J. Infrared and Millimeter Waves, 10, 907930, 1989.

Schandra, E., Physical Fundamentals of Remote Sensing, chapter 5, Splinger-Verlag, Berlin, 1986.

Takahashi, T. and F. Tohyama, Geomagnetic attitude measurement, Institute of Space and Astronautical Science Report no. 38, 65-68, 1998.

Tohyama, F., M. Ishido, and H. Fukunishi, Attitude determination of the Antarctic sounding rocket from magnetometer and horizon aspect sensor data, J. Geomag. Geoelectr., 40, 817-829, 1988.

N. Iwagami (e-mail: iwagami@eps.s.u-tokyo.ac.jp), S. Komada, and T. Takahashi 